\title{
LA ACTIVIDAD EN TERAPIA OCUPACIONAL Y LOS PROCESOS DE CONSTITUCIÓN DEL SUJETO EN DISPOSITIVOS DE SALUD MENTAL
}

\author{
ACTIVITY IN OCCUPATIONAL THERAPY AND PROCESSES OF CONSTITUTION OF \\ THE SUBJECT IN DEVICES MENTAL HEALTH
}

\section{Juliana Luz Czernik, Jesica María Sobrero, Lia Florencia Tomatis (')}

\begin{abstract}
Resumen:
Esta publicación corresponde a un Plan de Investigación sin trabajo de campo. Problematiza cómo la actividad en Terapia Ocupacional conforma procesos de constitución del sujeto en dispositivos de Salud Mental. Para responder a esta pregunta, se utilizan criterios que Michel Foucault maneja en el recorrido de sus obras. A modo de análisis, se entiende que la actividad en Terapia Ocupacional, puede ser planteada como posibilidad de movimiento y trasformación, lo cual se aproxima a la subjetivación; o bien las condiciones en las que se presenta la actividad pueden tender a la producción y repetición de modelos de subjetividad, quedando el sujeto objetivizado en esa lectura ajena. La publicación plantea una Fundamentación, un Estado del Arte, un Problema de investigación, Objetivos y un Diseño Metodológico. Respondiendo a un Tipo de Investigación Social, según Souza Minayo M. C. (1997), y un Tipo de Estudio DescriptivoExploratorio, según la clasificación de Sabulsky J. (2000).
\end{abstract}

\section{Palabras clave:}

Actividad en Terapia Ocupacional - Constitución del Sujeto - Dispositivos de Salud Mental.

\begin{abstract}
:
This publication pertains to a Research Plan without fieldwork. Questions how the activity conforms processes of subject formation in devices Mental Health. To answer this question, Michel Foucault criteria are used. As an analysis, it is understood that activity in Occupational Therapy, as an object of study and disciplinary intervention strategy, can be considered under conditions allowing that as a possibility of movement and transformation, enabling the subject to find "something" that has to do with himself, which approximates the subjectivation; or the conditions in which the activity is presented may tend to be constituted as an instrument of production and repetition of subjectivity models, being the subject reading object. The publication presents a Groundwork, State of the Art, Research Problem, Objectives and Design Methodology. It is a Type of Social Research, as Souza Minayo M. C. (1997), and Type of ExploratoryDescriptive Study, by classification of Sabulsky J. (2000).
\end{abstract}

\section{Keywords:}

Activity in Occupational Therapy - Constitution of the Subject - devices Mental Health.

Licenciadas en Terapia Ocupacional de la Universidad Nacional del Litoral, Santa Fe, Argentina. Esta producción corresponde a un extracto del Trabajo Final de Grado para la Licenciatura en Terapia Ocupacional.

Juliana Luz Czernik: juli_luzc@hotmail.com

Jesica María Sobrero: jesicasobrero_15@hotmail.com

Lia Florencia Tomatis: liatomatis@yahoo.com.ar 


\section{INTRODUCCIÓN}

¿Cómo comenzó nuestro interés por este trabajo de investigación?

Las experiencias transcurridas a lo largo de nuestra formación en diferentes materias teóricas y en el cursado de las prácticas profesionales, nos llevaron a preguntarnos por los distintos modos de intervención que se establecen en Terapia Ocupacional (de ahora en más T.O.) en el campo de la Salud Mental.

Creemos que la idea de intervención en dicha disciplina está directamente referida a la categoría actividad y supone una acción que, en el mejor de los casos, debe alcanzar efectos terapéuticos vinculados a la consideración de lo subjetivo. Pese a ello, percibíamos que el uso terapéutico de las actividades en la práctica de T.O. respondía generalmente a una función normalizadora de los sujetos, en tanto pretendía acercarlos a un "desempeño normal de sus funciones". Esto nos planteaba un interrogante: ¿cómo considerar lo subjetivo de las personas abordadas a partir de modos de intervención basados en la normalización?

Transitar por espacios que intentaban ser críticos con el quehacer del terapeuta ocupacional (de ahora en más t.o.), promovió en nosotras la experiencia de reflexionar sobre las posibilidades de objetivación y subjetivación que se instituyen a partir de la actividad, dándole fuerza a nuestro deseo de buscar alternativas para concebir lo terapéutico y comprender al sujeto.

¿Qué perspectiva pretendemos para este trabajo?

Creemos oportuno adoptar ciertos criterios que Foucault construye en el recorrido de sus obras al desarroIlar una historia de las problematizaciones, lo que él llama genealogía $\left({ }^{2}\right)$. En este caso lo que problematizaremos es ¿cómo la actividad en T.O. conforma procesos de constitución del sujeto en dispositivos de Salud Mental? Consideramos que esta perspectiva nos brinda categorías interesantes para reflexionar y analizar la temática de este trabajo.

2 Genealogía: Es una investigación histórica que se opone (...) a la unicidad del relato histórico y a la búsqueda del origen y que indaga, al contrario, en la "singularidad de los acontecimientos al margen de toda finalidad monótona". El método genealógico es una tentativa de desujeción de los saberes históricos, esto es, de hacerlos capaces de oposición y lucha contra "el orden del discurso", lo cual significa que la genealogía no busca sólo en el pasado la huella de acontecimientos singulares, sino que se plantea la cuestión de la posibilidad de los acontecimientos hoy en día (...) (Revel 2009, p. 71-72).
En primer lugar es importante dejar en claro que vamos a considerar a la actividad en T.O. en términos de lo que Foucault plantea como práctica discursiva, es decir, cómo saber ejercido (Britos M.P., 1994). Lo que permite indagar cómo se llevan a cabo las actividades, qué concepciones hay de ellas y desde qué posicionamientos son pensadas. Por lo pronto vemos que desde esta categoría, la actividad en T.O. siempre remite a un discurso que constituye un saber, permitiéndonos pensar que no es un "simple hacer" y así poder reflexionar sobre la coexistencia entre la teoría y la práctica.

Así como se ha expuesto en las palabras clave de esta producción, otra categoría fundamental para poder abordar la problemática que guía esta investigación, es la de constitución del sujeto, la cual alude a la reflexión llevada a cabo por Foucault sobre el proceso de constitución de la subjetividad a lo largo de la historia. Para ello el autor remite a dos instancias de análisis: 1) los modos de objetivación y 2) las prácticas de subjetivación.

La intención de este trabajo es vislumbrar cómo la actividad conforma procesos de constitución del sujeto en talleres de T.O. que pertenecen a instituciones de Salud Mental. Para poder pensar a los talleres en donde se llevará a cabo la investigación tomaremos otro aporte de Foucault: la categoría dispositivo. Dicha conceptualización remite a una "red de relaciones que se pueden establecer entre elementos heterogéneos: discursos, instituciones, arquitectura, reglamentos, leyes, medidas administrativas, enunciados científicos, proposiciones filosóficas, morales, filantrópicas, lo dicho y lo no dicho" (Castro 2004, p. 98). Deleuze (1990) establece que estas redes -que él supone constituidas por líneas- forman procesos siempre en desequilibrio y singulares. De acuerdo a dicho autor, podemos situar tres dimensiones que conforman al dispositivo:

$1^{\circ}$ Dimensión compuesta por líneas de visibilidad y de enunciación, Foucault entiende al dispositivo como una máquina para hacer, ver y hablar.

$2^{\circ}$ Dimensión compuesta por líneas de fuerza, las cuales pasan por todos los puntos de un dispositivo. Esta dimensión nos permite analizar las condiciones de poder que constituyen el dispositivo.

$3^{\circ}$ Dimensión del sí-mismo, compuesta por líneas de objetivación y subjetivación. Refiere a la producción de subjetividad dentro de un dispositivo. Es decir, a partir de esta dimensión podemos preguntarnos cómo un dispositivo hace posible la constitución subjetiva. Esto responde a la idea de Foucault acerca de que vivimos y actuamos en dispositivos y en ellos nos constituimos a través de procesos de objetivación y subjetivación. Así 
la constitución subjetiva se produciría en el interjuego de dos líneas: líneas de objetivación y líneas de subjetivación o líneas de fuga.

Plantear la noción de dispositivo habilita el camino para comprender complejamente a los talleres de T.O. en Salud Mental, como redes de relaciones entre diversos elementos que aparecen en ellos, configurando saberes, poderes y subjetividades. Dichos elementos se "materializan" en espacios denominados terapéuticos, donde se juegan posibilidades e imposibilidades, dichos y no dichos, elementos que se repiten y hacen serie, $y$ otros que se fracturan y hacen fuga.

Tomando entonces estas tres categorías fundamentales (práctica discursiva, constitución del sujeto y dispositivo) decimos que la actividad en TO como práctica discursiva forma parte de dispositivos de Salud Mental, por lo que está atravesada por las diferentes líneas del mismo y, particularmente esta producción, focalizará sobre las líneas de objetivación y subjetivación, vislumbrando cómo éstas se dan en la actividad.

\section{¿Cómo construimos nuestro problema de investigación?}

Para comenzar, vemos necesario analizar concepciones de actividad que plantean autoras de nuestra disciplina, partiendo de algunos interrogantes que posibilitaron reflexionar el objeto de estudio de este trabajo: ¿Qué concepciones se tiene sobre la actividad en T.O.? ¿Cómo es pensada? ¿Con qué objetivos y en qué condiciones se usa?

En principio, citamos unas palabras de Martínez Antón (1997), quien dice que "la Terapia Ocupacional se instala en el estudio del hombre en actividad y de la actividad del hombre como objeto fundamental" (p. 3). Tomando lo enunciado, la actividad y el hombre en vinculación constituyen nuestro eje disciplinar. En otra ocasión, plantea que la T.O. tiene como objetivo tratar las dificultades de los sujetos en el manejo de las actividades y ocupaciones, resaltando la función de estos artificios en el sostenimiento de la cultura (2000).

Por otra parte, Paganizzi (1997) expresa que en T.O. se toman las actividades como una de las estrategias para mejorar el rumbo de una determinada situación subjetiva, comunitaria o social, junto al paciente o grupo. A su vez, la autora manifiesta que la utilización de actividades siempre está fundamentada desde alguna teoría que necesariamente se halla ligada a concepcio- nes acerca del hombre, de la etiología de su padecer y de las estrategias para mitigar dicho sufrimiento.

Respondiendo al posicionamiento que adoptamos, y como ya hemos planteado, vamos a referirnos a la categoría actividad en términos de práctica discursiva, es decir, como "saber ejercido, como una red de enunciados y estrategias que se determinan recíprocamente" (Britos 1994, p. 10).

Teniendo en cuenta dicha definición, decimos entonces que en la actividad en T.O. hay un hacer y un saber que se vinculan y al mismo tiempo la conceptualización refiere a un conjunto de enunciados, y por tanto, a un discurso. Los discursos, según Malachevsky (2003), refieren a conjuntos de enunciados -que pueden tomar la forma de representaciones, creencias, teorías, modelos-que ordenan la realidad, generan prácticas sociales y enmarcan las conductas. Así, los discursos encierran supuestos sobre la subjetividad que pueden entenderse como "demandas": la adecuación de las respuestas por parte de los sujetos a las demandas del discurso, no solo implica consentir a ciertos modos de hacer o ver que el mismo propone, sino fundamentalmente, a una manera particular de configurarse como sujeto dentro de ese universo significante. De este modo, el discurso no se limita únicamente a producir significaciones sino que define modos de vincularse de los sujetos, estableciendo relaciones de poder y construyendo jerarquías.

A partir de lo dicho, estamos en condiciones de afirmar que las actividades en T.O. no son sólo prácticas, entendidas como simples quehaceres, sino que remiten a saberes discursivos, y como tales, éstos producen sentido, determinan prácticas sociales, enmarcan conductas, configuran subjetividades y modos de vinculación social. Las actividades como prácticas discursivas son objeto de estudio y estrategia de intervención disciplinar. Los t.o. producen sentido sobre ella, construyen discursos sociales que remiten a modos de intervenir, pero a la vez, la misma actividad genera efectos de sentido en los sujetos abordados y los acerca a instancias de constitución subjetiva.

En esta instancia creemos conveniente profundizar en las conceptualizaciones que Foucault plantea acerca de la constitución del sujeto en la trama histórica, haciendo referencia a un proceso de dos instancias: los modos de objetivación y las prácticas de subjetivación (Britos, 1994). El autor refiere a los procesos de constitución de un sujeto o una subjetividad, a partir de dos tipos de análisis:

1) Los modos o prácticas de objetivación: Solo se puede ser sujeto al objetivarse. 
En este primer análisis, ser sujeto para Foucault correspondería a estar sujeto en una red de relaciones de poder y control, entendiendo estos modos como prácticas que tienden a organizarnos a la manera de los objetos (Britos, 1994). De acuerdo a este análisis, Foucault presenta tres modos de objetivación:

- Primer modo de objetivación: prácticas epistémicas, son representadas por las Ciencias Humanas, quienes establecen regímenes de verdad, utilizando sus discursos como marcos en los que es posible problematizar, controlar, enderezar la vida de los individuos en la sociedad moderna (Britos, 1994).

- Segundo modo de objetivación: prácticas divisorias. Constituyen aquellas modalidades por las cuales se segrega a los individuos respecto de los demás o de sí mismos. Dichas prácticas responden a una lógica binaria que permite ordenar y clasificar a los hombres en tanto son locos o cuerdos, sanos o enfermos, honrados o criminales, normales o anormales (Britos, 1994).

- Tercer modo de objetivación: prácticas de sujeción. Son aquellos dispositivos que determinan al hombre desde fuera, haciéndolo objeto de saber y segregación en la propia relación consigo mismo, llevándolos luego a reconocerse en esas mismas objetivaciones (Britos, 1994).

Podemos decir que en esta instancia la lectura es unidireccional: los sujetos son hablados, es decir, sólo pueden ser nombrados y reconocidos en tanto se sitúan del lado del objeto (Britos, 1999).

2) El segundo análisis que realiza Foucault corresponde a las prácticas de subjetivación: La manera en que nos relacionamos con nosotros mismos, son técnicas de sí que nos permiten constituirnos como sujetos (Revel J., 2009).

En estas prácticas de subjetivación, el sujeto se constituye a sí mismo "a partir de una experiencia concreta de libertad y verdad que posibilita una manera particular de relación consigo mismo y con los demás" (Benassi et al., 2003, p. 21). Ser sujeto, en esta etapa de la obra de Foucault, se vincula al autogobierno o gobierno de sí y no a estar sujetos a un control externo. El sujeto ya no se constituye en torno a la ley y la prohibición, sino que tiene voluntad de conocerse a sí mismo, comparando su experiencia con lo verdadero y lo falso, lo normal y lo patológico. El sujeto es libre respecto de los códigos morales y, en esa experiencia de libertad, busca transformarse a sí mismo, haciendo de su vida una obra estética (Britos, 1994).

En relación a este segundo análisis, Foucault nos propone que la pregunta sobre quiénes somos habilita un movimiento que trata de rechazar una y otra vez las respuestas que vienen de afuera, hacer tajos y pliegues en la superficie de su lenguaje. El movimiento consiste en oponer a las definiciones que nos nombran, señales de algo que se escapa a esas enunciaciones. Pero, no se trata de pretender reconocer y hacer que los otros reconozcan esas señales, porque eso sería nuevamente objetivarlas. Más bien la subjetivación tiene que ver con el movimiento de hacer pliegues y repliegues en el terreno de las experiencias objetivadas. Se trata de ir traduciendo el lenguaje de "lo normal" de manera tal que se habiten otros significados, que permitan jugar otro juego (Britos, 1999).

Entonces podemos decir que todo movimiento no escapa a cierta objetivación pero, en la medida en que el discurso lo permita, la subjetivación introduce otros posibles, habilita la duda, la pregunta que no espera respuestas más apropiadas (Britos, 1999). Como Foucault expresa, la constitución del sujeto es un proceso en donde el sujeto se aproxima a la subjetivación en medio de objetivaciones. No obstante, cabe mencionar que el modo en que se planteen las prácticas discursivas abre mayores o menores posibilidades para la experiencia subjetivante.

En este último sentido, las actividades que se llevan a cabo como medio terapéutico en los talleres de T.O. pueden pensarse como prácticas que ofrezcan mayores posibilidades para la subjetivación con la oportunidad de que la persona pueda encontrar en la actividad que se le proponga "algo propio", la experiencia de preguntarse por sí mismo sin tener de antemano una respuesta que lo defina desde afuera y, en ese lugar de afirmación singular, el sujeto puede generar una marca con la que se incorpora a la propuesta terapéutica, lo cual conforma un trabajo de construcción de lo propio.

Para pensar en esto creemos pertinente citar a Martínez Antón (2000), intentando reflexionar sobre la actividad en T.O. conformando procesos de constitución del sujeto. La autora explicita que la ocupación "forma parte de la producción de subjetividad, deja marcas en el cuerpo y provoca malestares" (p. 11). Continúa diciendo que la participación en actividades y ocupaciones nos posiciona en un orden simbólico: nos da un lugar y organiza nuestra existencia como sujetos. Pero esta participación no es azarosa, está determinada por el lugar que 
se nos dio (desde los grupos sociales de pertenencia), por las condiciones de la época, contexto cultural, etc. y por nuestra propia respuesta como sujetos.

También Yujnovsky (2001) nos ayuda a comprender los procesos de constitución del sujeto que se dan en torno a la actividad en T.O., refiriendo a ella como "un conjunto de acciones con un fin y un ordenamiento propio, que produce efectos sobre la realidad y sobre quien hace" (p. 1). La autora manifiesta que cuando alguien hace se producen efectos, y entonces, podemos pensar la actividad como un medio terapéutico; pero las actividades de por sí no curan ni enferman, sino que lo terapéutico tiene que ver con las condiciones en las que se producen: discursos sobre el sujeto, sobre lo que se considera terapéutico y concepciones de salud y enfermedad; prácticas discursivas en relación a la planificación terapéutica, personas que participan en ella, dinámica institucional, disposición de los espacios físicos, etc. Por lo tanto, "no cualquier actividad, en cualquier contexto, ni con cualquier fin tiene efectos terapéuticos" (Yujnovsky 2001, p. 1). Vinculando estos aportes con la categoría dispositivo, vemos que las condiciones en las que se plantean las actividades refieren a la singularidad del dispositivo, a la disposición de sus líneas, a su conformación.

En este sentido, pensamos que la actividad puede ser planteada bajo condiciones que la habiliten como posibilidad de movimiento y trasformación, dando lugar a que el sujeto encuentre "algo" que tenga que ver con él mismo, lo cual se aproxima a la subjetivación; o bien las condiciones en las que se presenta la actividad pueden tender a que se constituya como instrumento de producción y repetición de modelos de subjetividad, quedando el sujeto objetivizado en esa lectura ajena. Por lo que nos resulta interesante problematizar, cómo la actividad en T.O. conforma procesos de constitución del sujeto en dispositivos de Salud Mental.

\section{MÉTOdo}

\section{Problema de Investigación}

¿Cómo la actividad en T.O. conforma procesos de constitución del sujeto en dispositivos de Salud Mental?

\section{Objetivos de Investigación}

\section{Objetivo general}

- Indagar cómo la actividad en Terapia Ocupacional conforma procesos de constitución del sujeto en dispositivos de Salud Mental.

- Objetivos específicos:

- Describir y analizar cómo se conforma cada dispositivo de Salud Mental seleccionado.

- Describir y analizar los discursos que aparecen en relación a la actividad en cada dispositivo de Salud Mental.

- Describir y analizar a la actividad conformando una práctica epistémica, divisoria y de sujeción en cada dispositivo de Salud Mental.

- Describir y analizar a la actividad como práctica de subjetivación en cada dispositivo de Salud Mental.

\section{Diseño Metodológico}

\section{Tipo de diseño: Investigación Social}

Souza Minayo M. C. (1997) expresa que el término Metodologías Cualitativas consagra una dificultad histórica de las teorías de posicionarse frente a la especificidad de lo social; también manifiesta que dicho término supone una afirmación de la cualidad contra la cantidad, a la cual se opone, resaltando la interdependencia entre los aspectos cuantificables y la vivencia significativa de la realidad objetiva en lo cotidiano. En su lugar propone la noción de Metodologías de Investigación Social, a la cual adherimos y empleamos en esta investigación.

La Investigación Social es capaz de incorporar la cuestión del significado y de la intencionalidad como inherentes a los actos, a las relaciones y a las estructuras sociales, siendo estas últimas tomadas como construcciones humanas significativas. Así como las teorías sociales, este tipo de investigación refleja posiciones frente a la realidad, momentos de la historia y de la dinámica social, preocupaciones e intereses de clases y de grupos determinados. Implica una actitud y una 
práctica teórica de constante búsqueda que define un proceso intrínsecamente inacabado y permanente. Requiere de parte del investigador actitudes fundamentales como la apertura, flexibilidad, capacidad de observación y de la interacción del grupo de investigadores con los actores sociales involucrados.

El tipo de estudio, según la clasificación de Sabulsky J. (2000), será descriptivo exploratorio, ya que se propone indagar, y para ello describir y analizar, cómo la actividad en T.O. conforma procesos de constitución del sujeto en dispositivos de Salud Mental. A su vez, es exploratorio porque es un problema poco estudiado por la disciplina en cuestión, teniendo como referencia el estado del arte antes mencionado. Lo que se procura desde este diseño es incrementar el conocimiento sobre las categorías abordadas, con el fin de generar nuevos aportes a la T.O. De este modo, este trabajo posibilitará sentar bases para posteriores estudios.

Es importante resaltar que esta producción no pretende la generalización de los resultados, por el contrario intenta profundizar sobre la problemática de investigación aquí planteada, particularmente en los dispositivos seleccionados. Además, al considerar lo que propone la categoría foucaultiana dispositivo sobre la singularidad que presentan los mismos, no es posible generalizar los resultados.

\section{Unidad de análisis}

Estudiaremos a la actividad propuesta en los talleres seleccionados, conformando procesos de constitución del sujeto.

\section{Muestra}

Criterios de selección de la muestra:

- Que el taller pertenezca a una institución de Salud Mental.

- Que las instituciones que alojen a los talleres seleccionados pertenezcan a dependencias privadas y públicas.

- Que el taller tenga modalidad ambulatoria.

- Que el taller seleccionado esté coordinado por un Terapeuta Ocupacional.
- Que la población que asista al taller sean jóvenes y adultos.

Para la realización de este estudio, se seleccionaron de modo no aleatorio (intencional), teniendo en cuenta los criterios nombrados anteriormente, tres instituciones de Salud Mental, escogiendo de cada una de ellas un taller específico que conformará la muestra para abordar la unidad de análisis. De las tres instituciones elegidas, dos son de dependencia privada de la ciudad de Santa Fe, y una posee dependencia pública y se encuentra en la ciudad de Paraná. Las mismas fueron seleccionadas intencionalmente porque nos resultó interesante conocer ambas modalidades (pública y privada), para analizar las formas en las que se presentan los dispositivos, es decir, los contextos, las políticas que los atraviesan, los actores que allí aparecen.

Creemos que la muestra se adecuará mejor a las categorías foucaultianas aquí consideradas, cuando implique cierta diversidad -modalidad pública y privada- y, fundamentalmente, una institución dependiente de políticas públicas, estatales. Teniendo en cuenta que Foucault realiza un complejo análisis de entidades macrosociales (cárceles, hospitales, fábricas, escuelas, etc.) y de políticas estatales al reflexionar sobre estas categorías.

A continuación se presentarán las instituciones con los talleres que fueron elegidos para este trabajo, conforme a los criterios de selección de la muestra. La fuente de la cual se obtuvo la información para presentarlas corresponde a informes institucionales de las mismas.

1. Centro de Rehabilitación Psicosocial "Rumbos", situado en Suipacha 2646, ciudad de Santa Fe. El taller seleccionado es el de Arte y Reciclado.

2. "Hospital Escuela de Salud Mental", situado en Ambrosetti y Rondeau, ciudad de Paraná, provincia de Entre Ríos. Dentro del Hospital de Día se eligió el Taller de Microemprendimiento.

3. "Vaivén. Espacios en movimiento" ubicada en calle Urquiza 2500, de la ciudad de Santa Fe. En este caso se seleccionará el Taller de las Palabras.

\section{Instrumentos de recolección de datos}

Consideramos que a partir de los instrumentos que se utilizarán para realizar este estudio -entrevistas, ob- 
servaciones participantes y revisiones de documentos y registros- podremos tener acceso a información útil que nos permitirá indagar cómo la actividad conforma procesos de constitución del sujeto. A su vez, los mismos guardan coherencia con el tipo de orientación elegida para el diseño metodológico.

Los instrumentos seleccionados nos posibilitarán conocer a los diferentes discursos y saberes que constituyen a la actividad en T.O., en tanto práctica discursiva. En este sentido, las entrevistas y la revisión de documentos brindarán datos importantes sobre lo discursivo, donde se tratará de visualizar cómo los enunciados sobre la actividad en T.O. ordenan la realidad del dispositivo, generan prácticas y enmarcan las conductas, para hacer hincapié en los procesos de constitución del sujeto que conforma la actividad. A su vez, a partir de las observaciones participantes conoceremos cómo esos discursos toman cuerpo en las prácticas discursivas. A continuación la cita nos ayuda a pensar en esto:

Las prácticas discursivas no son pura y simplemente modos de fabricación de discursos. Ellas toman cuerpo en el conjunto de las técnicas, de las instituciones, de los esquemas de comportamiento, de los tipos de transmisión y de difusión, en las formas pedagógicas que, a la vez, las imponen y las mantienen (Castro 2004, p. 94).

También, la observación participante nos acercará a lo no discursivo del dispositivo de Salud Mental, para arribar al objetivo de investigación. En este sentido, Foucault plantea que el dispositivo integra a las prácticas discursivas y a las no discursivas, y como objeto de análisis, la categoría aparece ante la necesidad de incluir a las prácticas no discursivas, lo cual implica considerar a las relaciones de poder (Castro, 2004).

Los instrumentos elegidos nos permitirán analizar y describir cómo se conforma cada dispositivo de Salud Mental seleccionado y, para este fin, se tomarán como eje las tres dimensiones de los mismos según Deleuze (1990): del saber, del poder y del sí mismo. También se considerará analizar las redes de relaciones entre los elementos heterogéneos que conforman cada dispositivo elegido (Castro, 2004).

Asimismo, los datos que se obtengan a partir de los instrumentos de recolección serán analizados considerando las conceptualizaciones que realiza Foucault sobre procesos de constitución del sujeto, describiendo cómo la actividad constituye una práctica epistémica, divisoria y de sujeción y, también, conforma una práctica de subjetivación.

Visto lo que se pretende indagar en este estudio, a continuación se detallarán los instrumentos utilizados para esta finalidad:

- Entrevista, considerada según Kahn y Cannell (citado en Souza Minayo, 1997) como una conversación entre dos, hecha por iniciativa del entrevistador, destinada a buscar informaciones pertinentes para un objeto de investigación.

Souza Minayo (1997) establece que lo que toma la entrevista para la recolección de datos es la posibilidad del habla, de ser reveladora de condiciones estructurantes, de sistemas de valores, normas y símbolos y -al mismo tiempo- tiene el poder de transmitir las representaciones de los grupos en ciertas condiciones sociales, históricas, económicas y culturales. El tipo de entrevista que se aplicará es la semiestructurada, según la clasificación de Honnigmann (citado en Souza Minayo, 1997), es la que combina preguntas cerradas (o estructuradas) y abiertas, donde el entrevistado tiene la posibilidad de discurrir por el tema propuesto, sin respuesta o condiciones prefijadas por el investigador pero dentro de una estructura regular de interrogantes o tópicos que sí son precisados por éste.

Creemos relevante que dicho instrumento se aplique a dos actores fundamentales en esta investigación: los t.o. coordinadores de los talleres y las personas que participan de los mismos.

La entrevista que se le realizará a los coordinadores de los talleres tendrá fundamentalmente la intención de preguntar acerca de cuatro puntos que consideramos principales para poder indagar cómo la actividad conforma procesos de constitución del sujeto en dispositivos de Salud Mental. Se investigará el discurso del t.o. en relación a:

1. La institución: objetivos institucionales, servicios brindados, estructura edilicia, personas que asisten. La intención de investigar sobre la institución entrevistando solo a los t.o. (y no a otros profesionales y directivos) tiene como finalidad rescatar el discurso de los mismos en relación a cómo lo institucional se vincula con la temática aquí planteada.

2. El taller: encuadre, objetivos, actividad, relaciones entre sujetos. 
3. Las personas que participan del taller: consideraciones sobre el sujeto, relación sujeto-actividad.

4. Sus intervenciones y posicionamientos teóricos: concepciones sobre salud/enfermedad, sujeto y terapias; concepciones sobre el trabajo del t.o.; evaluación; objetivos terapéuticos; modalidades y contenidos de los registros (sobre el sujeto, la dinámica del taller, acontecimientos clínicos, entre otros).

También se realizarán entrevistas a los jóvenes y adultos que participan de los tres talleres que conforman la muestra, a partir de esto se buscará indagar qué discursos transmiten respecto de las actividades llevadas a cabo en el taller y cómo se vinculan a las mismas, rescatando material útil para analizar el objetivo central de esta investigación.

- Observación participante: según Schwartz y Schwartz (citado en Souza Minayo, 1997), se refiere al proceso donde se mantiene la presencia del observador en una situación social, con la finalidad de realizar una investigación. El observador recoge datos y, como es parte del contexto observado, modifica y es modificado por este contexto. Para este fin, se realizará una guía que organice la observación y sea útil para recolectar información específicamente sobre los siguientes puntos:

1. Acerca de la institución: cómo se ve reflejado el discurso del t.o. acerca de los objetivos institucionales, cuáles son los servicios brindados, como estructura edilicia, dinámica institucional, personal de la institución, personas que asisten.

2. Acerca del taller: encuadre (día, horario, frecuencia, personas que participan, entre otros); modos de implementación de las actividades; estrategias terapéuticas, relaciones entre sujetos.

3. Acerca de las personas que participan del taller: relación de éstas con la actividad.

4. Acerca del terapeuta ocupacional: modos de evaluación y de intervención del terapeuta ocupacional.; modalidades y contenidos de los registros
Además, se utilizará un diario de campo en donde las investigadoras volcarán por escrito la información recogida durante las observaciones participantes y todos aquellos datos no explorados en las entrevistas como ser: la relación entre lo que se transmite en un determinado discurso y lo que se hace en la práctica, los comportamientos, las conversaciones, entre otras.

- Revisión de documentos y diferentes registros: Los documentos que se tendrán en cuenta serán los siguientes: historias clínicas; informes de evolución, de evaluación, de ingreso o alta; crónicas; planes de tratamiento o propuesta de trabajo; epicrisis; bibliografía consultada por profesionales; boletines informativos; folletos institucionales; pizarras; paneles o carteles informativos y libros de acta.

La revisión de estos documentos nos permitirá vislumbrar información relevante para averiguar: los objetivos y estrategias terapéuticas que se persiguen; los modos de evaluación que se implementan; las actividades que se proponen y la información que se registra en relación al sujeto. También se buscará conocer cuáles son los posicionamientos o modelos teóricos que se sostienen tanto por la institución (profesionales y actores que la conforman) como por el terapeuta ocupacional. Como antes hemos dicho, la búsqueda de información sobre lo institucional y el análisis de la misma, siempre nos va a remitir a investigar cómo la actividad en terapia ocupacional conforma procesos de constitución del sujeto, por lo que no se intentará profundizar sobre los aspectos institucionales más allá de este objetivo.

En relación al tratamiento de los datos obtenidos mediante los instrumentos anteriormente citados, se realizará un análisis de los mismos, con el fin de obtener elementos que nos permitan elaborar aproximaciones a nuestro problema de investigación. Este análisis se llevará a cabo mediante lo que Deleuze (1990) Ilama cartografiar, esto sería tomar los enunciados recabados e ir disponiéndolos en series enunciativas, en donde se detectarán aquellas series que por aparecer reiteradamente serán significativas y generarán una cierta inteligibilidad sobre la problemática de investigación aquí planteada. En este sentido, la cartografía podrá entenderse como un operador epistémico, es decir, como el elemento que nos permitirá construir conocimiento acerca del objeto de estudio de esta investigación. 


\section{Discusión}

Al realizar una revisión de las producciones sobre la temática abordada, no se han encontrado, dentro de la disciplina T.O., antecedentes de investigación que analicen la relación entre las palabras claves que guían esta producción: actividad en T.O. y constitución del sujeto, motivo por el cual consideramos que este tema merece ser investigado. Sin embargo, se hallaron en nuestra disciplina, trabajos de investigación y artículos que aportan antecedentes relacionados a la problemática aquí planteada. A continuación haremos mención de los mismos teniendo en cuenta la cronología en la que se realizaron.

En el año 2003, las licenciadas Benassi J., Gómez J. y Rosciani P., publican su Trabajo Final de Grado para la Lic. en T.O. de la Universidad Nacional del Litoral, de la ciudad de Santa Fe, Argentina; titulado "Prácticas discursivas y dispositivos de internación en salud mental. La problemática de la Cronicidad". En el mismo investigan cómo se construye la cronicidad como objeto de conocimiento y práctica disciplinar; a partir de analizar prácticas discursivas, proponen arribar a los modos de objetivación de la misma. Desde claves teóricas foucaultianas, se indaga sobre la cronicidad en dispositivos de internación de la ciudad de Santa Fe, en sus dos modalidades: pública y privada. El aporte de esta producción, radica en la importancia de que el terapeuta ocupacional. se cuestione la cronicidad y sus prácticas en torno a ella, revirtiendo la lógica de la "imposibilidad" que se le suele otorgar a su abordaje y optando por un diseño de intervención "positivo", que tome como punto de partida lo que el paciente "puede" y a partir de allí arme la racionalidad de su práctica.

De la investigación anteriormente mencionada, hemos tomado como aporte el análisis que sus autoras han realizado sobre los modos en que se objetiviza la cronicidad, vislumbrando cómo las prácticas discursivas en Salud Mental pueden conformar procesos de constitución del sujeto. Además, las investigadoras se han planteado si la T.O. es un servicio para la producción de sujetos o para la construcción subjetiva, lo cual fue un interrogante clave para analizar las posibilidades que habilita la actividad en T.O. en relación a la constitución del sujeto.

En el año 2007, la terapeuta ocupacional, Carrasco J. publica la siguiente producción para obtener el título de Magister en Investigación en Psicología Social de la Universidad Atónoma de Barcelona, España: "Las metáforas de la Terapia Ocupacional: una aproximación a formas de gubernamentalidad ( )". Esta investigación es el resultado de un ejercicio crítico y reflexivo en el que se intenta desentrañar las formas de gobierno actuales que validan y dan vida a la T.O; para esto plantea que dicha disciplina ha delimitado un saber que responde a las diferentes racionalidades de gobierno, creando subjetividades deseables y generando técnicas coherentes con esas mentalidades. La autora postula que la T.O. va a delimitar un campo de gobierno: la ocupación humana como medio y fin, validando una determinada forma de intervención. Para poder estudiar tal situación y con el fin de acotar el ámbito de este estudio, escoge un modelo teórico y práctico propio de la T.O.: El Modelo de Ocupación Humana de Gary Kielhofner, por ser uno de los referentes más importantes en la actualidad para terapia ocupacional.

Creemos importante considerar este estudio como antecedente, teniendo en cuenta que la autora plantea que las formulaciones, los conceptos y las ideas de este modelo se han explorado como discursos instituidos como "verdaderos" en lo referente al sujeto deseable, como resultado del ejercicio de relaciones de poder y de resistencias, configurando un saber y un poder y con él una forma de gubernamentalidad. Bajo estas formas de gobierno se pueden vislumbrar ciertos modos de re-producir formas de intervenir desde T.O., las mismas tienen en cuenta las estrategias de un modelo y así se aproximan a una forma particular de constitución del sujeto.

En el año 2009, la terapeuta ocupacional Gutiérrez P. y el psicólogo Pujol Tarrés J., publicaron en el libro de Actas del XI Congreso Español de Psicología Social el siguiente artículo: "Constitución de sujeto en la práctica de la Terapia Ocupacional". Este trabajo adopta una perspectiva foucaultiana al considerar un análisis genealógico y gubernamental con el fin de identificar las concepciones de sujeto y de intervención que atraviesan las prácticas actuales en T.O. Los autores plantean que el ideal de dirigir a la persona hacia una actividad que la auto -realice se transforma en adaptar al sujeto a un contexto socio- productivo concreto; por lo tanto, se promueve el desarrollo de habilidades funcionales a la vez que se pierde el énfasis en las construcciones de significado de la persona en términos de sentido vital y autorrealizativo. Asimismo, expresan que ocurre a nivel 
ocupacional lo que ocurre a nivel médico respecto a la discapacidad: es aceptable intervenir médicamente sobre el cuerpo discapacitado para acercarlo a la "normalidad", generando a su vez, una comprensión individualizada de la problemática de esta persona, entendiendo que la causa de su problemática está en su propio cuerpo, limitando así, una comprensión compleja de la situación.

Dicho trabajo nos permitió pensar sobre la práctica profesional de los terapeutas ocupacionales, los supuestos con los que trabaja y sus objetivos de intervención; abriéndonos el sendero hacia la reflexión sobre las condiciones para la constitución del sujeto que la práctica puede potenciar. Además pone en foco las lógicas reproductoras de discursos médicos, cuestionando este tipo de abordajes y contextualizando a la práctica dentro de los procesos de gubernamentalidad para poder entender qué nociones de sujeto se hacen visibles en la sociedad.

También en el año 2011 la terapeuta ocupacional Gutiérrez P. publica su investigación para la graduación en el Doctorado en Psicología Social de la Universidad Autónoma de Barcelona; el mismo se titula: "Terapia Ocupacional: Una disciplina para la autonomía. Prácticas y discursos de Gubernamentalidad y subjetivación en torno a una ciencia emergente". La intención de este trabajo, es hacer dialogar a los diferentes agentes (principalmente terapeutas ocupacionales y personas con diversidad funcional) acerca de las formas de gobierno (racionalidades políticas y tecnologías de gobierno) y subjetivación presentes en las prácticas terapéuticas de Gutiérrez, analiza que dicha disciplina, al haberse centrado hasta ahora en la concepción de sujeto activo, autónomo e independiente, ha limitado la intervención, la ha vuelto funcional al modelo médico. En este sentido, la recuperación funcional del cuerpo es prioridad por sobre los deseos y aspiraciones de las personas, potenciando la figuración de un "sujeto ocupacional fragmentado", cuyas partes son potenciadas para el logro de su independencia, optimizando sus habilidades y el desarrollo de destrezas que serán claves para la formación de "ciudadanos activos" y ajustados a las normas y/o estándares de funcionamiento, que contribuyen a la construcción de cuerpos y subjetividades discapacitadas.

Esta investigación nos ha sido útil para reflexionar sobre los posibles saberes instaurados en la T.O. y, en consecuencia, sobre las prácticas profesionales que se despliegan de la misma. Además nos posibilitó cuestionarnos sobre las nociones de sujeto que tomamos en nuestra formación como terapeuta ocupacional y las posibles hipótesis por las que se interviene buscando un sujeto activo, sano, normal, dejando por fuera lo que la persona expresa, desea, busca. Creemos que, si nos cuestionamos la idea sobre lo ocupacional como una mera reproducción de subjetividades, podremos pensar en la singularidad de cada sujeto en su hacer. Como resultado de la reflexión de esta investigación hemos conocido que hay quienes también están comenzando a preguntarse por este tipo de planteos.

\section{ReferenCias BibliográficAs}

\section{Libros:}

Abbate, F.; Páez, P. (2006). Gilles Deleuze para principiantes. Buenos Aires: Era Naciente.

Bericat, E. (1998). La integración de los métodos cuantitativos y cualitativos en la investigación social: Significado y medida. Barcelona: Ariel.

Britos, M. P. (1994). Michel Foucault: La constitución del sujeto. Éticas del siglo. Asociación argentina de investigaciones éticas. Consejo regional de Santa Fe (pp. 9-22). Rosario, Santa Fe: Tekhne Impresión.

Castro, E. (2004). El vocabulario de Michel Foucault: un recorrido alfabético por los temas, conceptos y autores. Bernal: Universidad Nacional de Quilmes.

Deleuze, G. (1986). Foucault. Barcelona, España: Editorial Paidós Ibérica.

Deleuze, G. (1990). V.V.A.A. Michael Foucault, filósofo. "¿Qué es un dispositivo?" Barcelona, España: Editorial Gedisa.

Foucault, M. (1999). Historia de la sexualidad "1. La voluntad del Saber". España: $27^{a}$ Edición Siglo Veintiuno, Editores Madrid.

Foucault, M. (2002). Vigilar y castigar. Nacimiento de la prisión. Buenos Aires: Siglo veintiuno, Editores Argentina.

Foucault, M. (1992). El orden del discurso. Buenos Aires: Tusquets Editores.

Foucault, M. (2008). La arqueología del saber. España: Ediciones Siglo Veintiuno, Editores Madrid.

Paganizzi, L. (1997). Actividad: Lenguaje particular. Buenos Aires: Calamús.

Revel, J. (2009). El vocabulario de Michel Foucault. Buenos Aires: Nueva Visión.

Rocha Medeiros, M. H. (2008). Terapia Ocupacional. Un enfoque epistemológico y social. Santa Fe: Ediciones UNL.

Souza Minayo, C. (1997). El desafío del conocimiento. Investigación cualitativa en Salud. Buenos Aires: Editorial Lugar. 


\section{Apuntes de Cátedra:}

Benassi, J. (2004). Filmina clase diez: Paradigma Positivista: Actividad Ejercicio-Actividad Producción/Paradigma Interpretativo: Actividad Expresión. Apunte de cátedra: Estética I. Carrera: Lic. en Terapia Ocupacional. Escuela Superior de Sanidad, FBCB, UNL. Santa Fe.

Malachevsky, J. (2004). Poder y subjetividad. Apunte de cátedra: Intervención en dispositivos de salud mental. Carrera: Lic. en Terapia Ocupacional. Escuela Superior de Sanidad, FBCB, UNL. Santa Fe.

Malachevsky, J. (2003). Dispositivos. Apunte de cátedra: Intervención en dispositivos de salud mental. Carrera: Lic. en Terapia Ocupacional. Escuela Superior de Sanidad, FBCB, UNL. Santa Fe.

Malachevsky, J. (2005). El sujeto y los discursos. Sobre los impactos discursivos en la subjetividad. Apunte de cátedra: Intervención en dispositivos de salud mental. Carrera: Lic. en Terapia Ocupacional. Escuela Superior de Sanidad, FBCB, UNL. Santa Fe.

Martínez, Antón, R. (1997). Análisis funcional y análisis ocupacional. Apuntes de Cátedra: Teoría y Técnica de Terapia Ocupacional I. Carrera de Terapia Ocupacional. Universidad de Buenos Aires.

Yujnovsky, N. (2001). ¿Por qué se hacen actividades en el Hospital de Día? Aporte para el ateneo del Hospital de Día "Caminos". Hospital "Antonio L. Roballos". Paraná, Entre Ríos, Argentina.

\section{Artículos de Revistas:}

Mantero, P. \& Soria, M. (1997). La realidad de los medios terapéuticos: acerca de la actividad humana y lo terapéutico. Materia Prima, Año 2, Número 5, 21-23. Buenos Aires.

Martínez Antón, R. (2000). La Actividad y la Ocupación. Materia Prima, Sección: De Producción Nacional. Año 4 Número 13, $p$ 9- 12. Buenos Aires.

\section{Tesis de Grado o Post-Grado:}

Benassi J., Gómez J. y Rosciani P. (2003). "Prácticas discursivas y dispositivos de internación en salud mental. La problemática de la Cronicidad". Trabajo Final de Grado para la obtención del título de Licenciada en Terapia Ocupacional, Universidad Nacional del Litoral, Facultad de Bioquímica y Ciencias Biológicas, Escuela Superior de Sanidad, Santa Fe, Argentina.

Carrasco, J. (2007). "Las metáforas de la Terapia Ocupacional: una aproximación a formas de gubernamentalidad". Trabajo Final de Grado para obtener el título de Magíster en Investigación en Psicología Social de la Universidad Autónoma de Barcelona,
España. (En línea). Disponible: http://psicologiasocial.uab.es/ fic/en/node/136.

Gutiérrez, P. (2011). "Terapia Ocupacional: una disciplina para la autonomía. Prácticas y discursos de gubernamentalidad y subjetivación en torno a una ciencia emergente". Tesis doctoral, Universidad Autónoma de Barcelona, Departamento: Psicología Social, España. (En línea). Disponible: biblioteca de comunicación y hemeroteca general uab 2011gutieterap.pdf.

\section{Webgrafía:}

Boletín Oficial del Estado. Ministerio de Salud de la Nación, Dirección Nacional de Salud Mental y Adicciones (2010). "Se aprobó la Ley Nacional de Salud Mental". (En línea). Disponible en:

http://xa.yimg.com/kq/groups/18213827/537517389/name/boletin

Gutiérrez, P. \& Pujol Tarrés, J. (2009). "Constitución de sujeto en la práctica de la Terapia Ocupacional". (En línea). Disponible: https://www.ucursos.cl/medicina/2009/0/INVETOUCH1/1/ material_docente/previsualizar?id_material $=257172$

Ley 26.657. Derecho a la Protección de la Salud Mental. Disposiciones complementarias (2010). (En línea). Disponible: http://infoleg. mecon.gov.ar/infolegInternet/anexos/175000-179999/175977/ norma.htm

Lipcovich, P. (2010, noviembre 26). "Un cambio de paradigma en la salud mental. Ya es ley el proyecto que impulsa la desmanicomialización y limita las internaciones compulsivas". Diario Página 12. (En línea). Disponible en:

http://www.pagina12.com.ar/diario/sociedad/3-157578-2010-11-26. $\mathrm{html}$

Manual de Normas APA (American Psychological Association), (2002) (En línea). Disponible en: http://lia.unet.edu.ve/ant/EstiloAPA.htm

\section{Otras Fuentes:}

Informes institucionales de: Fundación "Convivir", Hospital "Dr. Antonio L. Roballos" y "Vaivén. Espacios en movimiento".

Foucault, M. (1992) "La poussiére et les nuages", en Dits et Écrits, T IV. París, Gallimard. 